\title{
Suffering at the end of life: A systematic review
}

\section{Limonero, Joaquín T.11, Casellas-Grau, Anna²., Gómez-Romero, María José1,3, Maté-Méndez, Jorge ${ }^{1,4}$, Mateo Ortega, Dolors ${ }^{1,5}$, Cladellas-Pros, Ramon', Álvarez Maria1, Montes Hidalgo, Javier ${ }^{6}$, Tomás-Sábado, Joaquín ${ }^{6}$, Gil Moncayo, Francisco ${ }^{1,7}$}

${ }^{1}$ Universitat Autònoma de Barcelona, Stress and Health Research Group. Faculty of Psychology, Bellaterra (Cerdanyola del Vallès), Spain; ${ }^{2}$ Catalan Institute of Oncology, Psychosocial Observatory in Cancer, L'Hospitalet de Llobregat, Spain; ${ }^{3}$ Egarsat, Mutua Colaboradora con la Seguridad Social no 276, Psychology Unit, Terrassa, Spain; 4 Catalan Institute of Oncology, Psycho-oncology Care Unit, L'Hospitalet de Llobregat, Spain; ${ }^{5}$ Consorci Sanitari de Terrassa, Palliative Care Unit, Terrassa, Spain; Gimbernat University Schools. Universitat Autònoma de Barcelona, Research and Scientific Communication Unit, Sant Cugat del Vallès, Spain, ${ }^{7}$ Catalan Institute of Oncology, Psychooncology Unit. Psychosocial Observatory in Cancer, L'Hospitalet de Llobregat, Spain.

\section{BACKGROUND}

There is currently a considerable amount of studies exploring suffering among patients with terminal illnesses. To date, no systematic reviews have been performed exploring which are the factors that trigger suffering at the end of life in patients diagnosed with chronic illnesses.

\section{$A / M$}

We aim to systematically explore, synthesize, and reach a consensus about those factors which trigger end-of-life suffering in patients diagnosed with advanced chronic illnesses.

\section{METHODS}

The four major databases in the field (PsycINFO, Pubmed, Web of Science, Scopus) were explored using relevant terms that enabled the access to relevant literature in accordance to the review focus: 'suffering', 'emotional distress', 'end-of-life', 'advanced illness' and 'terminal illness'. PRISMA guidelines were followed, screening the articles by relevance through their title, abstract, and full text. The abstracts of the identified records were screened for relevance. The quality of the studies will be assessed using the critical appraisal tools according Kmet, Lee \& cook (2004) related to Standard Quality Assessment Criteria for Evaluating Primary Research Papers. This study is registered in PROSPERO 2019 CRD42019124419

\section{RESULTS}

$\rightarrow$ From the 1,978 results obtained from databases after removing duplicates, 92 articles finally met the inclusion criteria. Most studies used quantitative methods to assess their results, except for 22 that used qualitative methodology (23.9\%), and 10 which used mixed-methods $(10.8 \%)$.

$\rightarrow$ In general, studies reported few relationships between sociodemographic characteristics and suffering at the end of life (e.g. age, gender, marital status, social support, ethnics-race, socioeconomic status, education levels)

$\rightarrow$ Preliminary results show that the most relevant factors that generate suffering among patients at the end of life can be grouped in three big dimensions:

Physical characteristics (e.g. pain, fatigue, dyspnea, proximity to death, bad physical functioning)

Suffering and psychological responses (e.g. emotions related to coping strategies and illness situation in a negative way -lack of acceptance, denial, depression, hopelessness; fear of death; or in positive way positive: outlook, self-distraction, active coping, positive reframing, social support

Suffering and spiritual and existential issues (e.g. religious fellowships, compromised dignity, lack of meaning in life, being a burden to others, unfinished business, being in pace, faith )

\section{DISCUSSION}

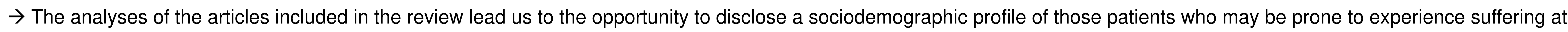
the end of life (young women with both low social support as well low economic status).

$\rightarrow$ This systematic review states the relevance of the physical dimension as a big source of suffering, especially for pain, dyspnea, and fatigue.

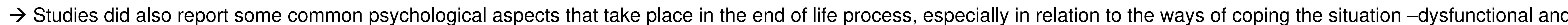
constructive styles- and psycho-emotional characteristics, like personal history of mood disorders, self-esteem, or emotional well-being.

$\rightarrow$ Different aspects of existential issues were explored, including those related to the sense of one's life (meaning, hopelessness, helplessness, demoralization, and lack of life satisfaction); those related to significant others (sense of being a burden to others, unfinished business), and its consequent wish to hasten death; and those related to one's spirituality, peace, or religiousness.

$\rightarrow$ Clinical implications: Results from this systematic review highlight the importance of taking into account the individual characteristics of each patient in terms of their sociodemographical, physical, psychological, spiritual, and adjustment to their situation, together with their style of coping strategies. 